ISSN 0103-8478

\title{
EFEITOS DA QUEIMA SEGUIDA DE PASTEJO OU DIFERIMENTO SOBRE O RESÍDUO, TEMPERATURA DO SOLO E MESOFAUNA DE UMA PASTAGEM NATURAL ${ }^{1}$
}

\author{
EFFECTS OF BURNING FOLLOWED BY GRAZING OR DEFERRING ON RESIDUAL \\ DRY MATTER, SOIL TEMPERATURE AND MESOFAUNA OF A NATURAL PASTURE
}

\author{
Paulo Rogério Viegas Damé ${ }^{2}$ Fernando Luiz Ferreira de Quadros ${ }^{3} \quad$ Cesar Eduardo Bicca Kersting $^{4}$ \\ José Pedro Pereira Trindade $^{5} \quad$ Zaida Inês Antoniolli $^{6}$
}

\section{RESUMO}

Foi realizado um experimento no Departamento de Zootecnia da UFSM, Santa Maria, RS, Brasil, com o objetivo de estimar os efeitos do fogo associado ao pastejo, com ou sem periodo de diferimento após a queima, sobre o resíduo e mesofauna edáfica de uma pastagem natural. Foi observada, também, o efeito do fogo sobre a variação da temperatura do solo. $O$ experimento foi instalado no inverno de 1992 e os dados foram coletados no periodo de maio de 1993 a junho de 1994. Os tratamentos foram: queimado em 17/09/92 e pastejado; queimado em 17/09/92, diferido, requeimado em 05/09/93 e pastejado; não queimado e pastejado em 1992, queimado em 22/06/93 e diferido. Foi constatado que o fogo diminui o resíduo da pastagem e essa redução é maior ainda quando há pastejo. Não foram observadas alterações significativas na temperatura do solo, devido à intensidade do fogo ter sido baixa. A queima e o pastejo não apresentaram efeitos significativos sobre a população de colêmbolos $e$ ácaros do solo.
Palavras-chave: queima, diferimento, pastagem natural, residuo, temperatura do solo, mesofauna do solo.

\section{SUMMARY}

This study was carried out to evaluate the effects of burning associated with grazing and burning with deferring periods, on residual dry matter, soil temperature and mesofauna composition of a natural pasture. The experiment was conducted at the Animal Science Departament, UFSM, Santa Maria, RS, Brasil. The trial was initiated in the Winter of 1992 and data were collected from May, 1993 to June, 1994. Treatments consisted of: burned in 9/17/92 and grazed; burned in 9/17/92 and deferred. reburned in 9/5/93 and grazed; not burned in 1992 and grazed, burned in 6/22/93 and deferred. Burning reduced dry matter residue and the reduction was more pronounced when burning was followed by grazing. Soil temperature was not affected by burning mainly because its intensity was low. Soil mesofauna was not significantly affected by the different treatments tested.

\footnotetext{
${ }^{1}$ Parte da Dissertação de Mestrado apresentada pelo primeiro autor para obtenção do grau de Mestre em Zootecnia, Universidade Federal de Santa Maria (UFSM), Santa Maria, RS. Trabalho executado com auxílio financeiro da FAPERGS e CNPq.

${ }^{2}$ Zootecnista, Pós-graduando em Zootecnia, UFSM, Bolsista do CNPq, Rua Quinze de Novembro, 1047, apto. 402, 96015-000, Pelotas, RS. Autor para correspondência.

${ }^{3}$ Engenheiro Agrônomo, Mestre, Professor Assistente, Departamento de Zootecnia, Centro de Ciências Rurais (CCR), UFSM

${ }^{4}$ Engenheiro Agrônomo, Pós-graduando em Zootecnia, UFSM, Bolsista do CNPq.

$\mathbf{5}$ Acadêmico do Curso de Agronomia, UFSM, Bolsista de Iniciação Científica da FAPERGS

${ }^{6}$ Bióloga, Mestre, Professora Assistente, Departamento de Solos, CCR, UFSM
} 
Key words: burning, deferring, natural pasture, residue, soil temperature, soil mesofauna.

\section{INTRODUÇÃO}

Existe controvérsia sobre a função do fogo no manejo de pastagens (CORRÊA \& ARONOVICH, 1979), considerando que a maioria das pastagens do mundo tem sido alterada e debilitada pelo uso excessivo do fogo e pelo sobrepastoreio (VOGL, 1974). No Rio Grande do Sul, a queima anual das pastagens naturais é usual, sendo uma das consequiências da taxa de lotação desvinculada do rítmo produtivo estacional da pastagem.

A comunidade de plantas de uma pastagem protege o solo da erosão hídrica e eólica, fixa nitrogênio no sistema e melhora as características físicas do solo. Estas melhorias nas condições do solo são o resultado das interações entre o sistema radicular das plantas, microorganismos e o solo (BARNES \& TAYLOR, 1985).

A cobertura vegetal, matéria orgânica, textura e umidade do solo e topografia influem diretamente na distribuição da sua fauna. Existe também, de modo geral, uma relação direta entre a fertilidade e a fauna do solo (RAPOPORT, 1968), em função do aporte de alimento.

Os organismos do solo são sensíveis às mudanças de estação, umidade, nutrientes, fatores bióticos e temperatura (AHLGREN, 1974). A interferência na temperatura do solo pode ser direta, pelo aumento do calor no momento da queima, ou indireta, pelo aumento nas flutuações térmicas em função da destruição da vegetação. $O$ estudo desses fatores é importante no monitoramento das alterações causadas pelas práticas pastoris sobre a vegetação e mesofauna do solo. Este trabalho teve o objetivo de estimar a influência da queima associada ao pastejo, com ou sem período de diferimento após o fogo, sobre o resíduo e mesofauna edáfica de uma pastagem natural e medir as variações da temperatura do solo durante a queima.

\section{MATERIAIS E MÉTODOS}

O experimento foi conduzido no Departamento de Zootecnia da Universidade Federal de Santa Maria, Santa Maria, RS, Brasil. O clima do local, situado na região fisiográfica da Depressão Central, é do tipo "Cfa" pela classificação de Köeppen (MORENO, 1961). O solo é um Brunizem Hidromórfico, pertencente à unidade de mapeamento Santa
Maria. A área experimental, utilizada sob pastejo contínuo, era formada por macegas entremeadas por uma vegetação rasteira, com predominância de gramíneas, como Andropogon lateralis, Eragrostis plana, Axonopus affinis, Paspalum notatum e $P$. pumilum. O delineamento experimental foi de blocos ao acaso, com três repetições, utilizando parcelas de $10 \mathrm{~m} \times 10 \mathrm{~m}$. O experimento teve início em 1992 e a descrição dos tratamentos está na Tabela 1.

Tabela 1. Descrição dos tratamentos (T) aplicados em uma área com pastagem natural. UFSM, Santa Maria, RS.

\begin{tabular}{llll}
\hline Datas e periodos & $\mathrm{T}_{1}$ & $\mathrm{~T}_{2}$ & $\mathrm{~T}_{3}$ \\
\hline $17 / 09 / 92$ & $\mathrm{Q}$ & $\mathrm{Q}$ & - \\
$17 / 09 / 92$ a $12 / 11 / 92$ & $\mathrm{P}$ & $\mathrm{D}$ & $\mathrm{P}$ \\
$13 / 11 / 92$ a $31 / 03 / 93$ & $\mathrm{P}$ & $\mathrm{P}$ & $\mathrm{P}$ \\
$01 / 04 / 93$ a $22 / 06 / 93$ & $\mathrm{D}$ & $\mathrm{D}$ & $\mathrm{D}$ \\
$22 / 06 / 93$ & - & - & $\mathrm{Q}$ \\
$23 / 06 / 93$ a $05 / 09 / 93$ & $\mathrm{D}$ & $\mathrm{D}$ & $\mathrm{D}$ \\
$05 / 09 / 93$ & - & $\mathrm{Q}$ & - \\
$06 / 09 / 93$ a $06 / 11 / 93$ & $\mathrm{P}$ & $\mathrm{P}$ & $\mathrm{D}$ \\
$07 / 11 / 93$ a $06 / 94$ & $\mathrm{P}$ & $\mathrm{P}$ & $\mathrm{P}$ \\
& & & \\
\hline
\end{tabular}

$\mathrm{Q}=$ queima $; \mathrm{P}=$ pastejo $; \mathrm{D}=$ diferimento.

As estimativas do resíduo da pastagem foram feitas com a utilização do Sistema Computacional BOTANAL (TOTHILL et al., 1978), modificado por COSTA \& GARDNER (1984). O resíduo foi definido como a quantidade de material vegetal aéreo presente em um determinado momento. Os dados do resíduo foram coletados em quadrados com $0,5 \mathrm{~m}$ de lado e os resultados foram expressos $\mathrm{em} \mathrm{kg} / \mathrm{ha}$ de matéria seca (MS). A temperatura do solo foi monitorada na superfície, a 5 e a $10 \mathrm{~cm}$ de profundidade, no início e no final da queima, com termômetro digital. Para a coleta da mesofauna do solo, foram instaladas três trampas Tretzel (TRETZEL, 1955) por parcela, antes e depois de cada queima e, posteriormente à queima, de forma estacional, ficando no campo de 4 a 5 dias.

Os dados foram submetidos a análise de variância e, quando houve diferenças significativas, as médias dos tratamentos foram comparadas pelo teste de Tukey, em um nível de significância de $5 \%$. Os dados de colèmbolos e ácaros sofreram transformação logarítmica. 


\section{RESULTADOS E DISCUSSÃO}

\section{Resíduo da pastagem}

O menor resíduo apresentado pelo $\mathrm{T}_{1}$, em 17/05/93, foi decorrente da queima seguida de pastejo (Tabela 2). No $T_{2}$, o resíduo foi recuperado através do diferimento da pastagem. No $\mathrm{T}_{3}$, o resíduo foi significativamente diminuído pela queima de 22/06/93, conforme pode ser observado em 27/08/93. Em 19/05/94, o $T_{3}$ apresentou maior média, possivelmente em função do efeito residual do diferimento na primavera, propiciando melhores condições de crescimento às plantas no outono. Pode ter ocorrido, também, menor consumo de forragem pelos animais, em relação às outras áreas, decorrente do engrossamento da pastagem pelo diferimento.

Tabela 2. Resíduo de matéria seca $(\mathrm{kg} / \mathrm{ha})$ de uma pastagem natural submetida aos efeitos do fogo e pastejo. Os dados estão expressos em matéria parcialmente seca, com uma média de $90,5 \%$ de matéria totalmente seca. UFSM, Santa Maria, RS.

\begin{tabular}{lccc}
\hline Datas & $\mathrm{T}_{1}$ & $\mathrm{~T}_{2}$ & $\mathrm{~T}_{3}$ \\
\hline $17 / 05 / 93$ & $1398 \mathrm{~b}^{*}$ & $2078 \mathrm{a}$ & $1997 \mathrm{a}$ \\
$27 / 08 / 93$ & $1789 \mathrm{~b}$ & $2402 \mathrm{a}$ & $1575 \mathrm{~b}$ \\
$11 / 12 / 93$ & $1156 \mathrm{a}$ & $1156 \mathrm{a}$ & $1345 \mathrm{a}$ \\
$05 / 03 / 94$ & $1239 \mathrm{a}$ & $1184 \mathrm{a}$ & $1450 \mathrm{a}$ \\
$19 / 05 / 94$ & $1384 \mathrm{~b}$ & $1364 \mathrm{~b}$ & $1968 \mathrm{a}$ \\
& & & \\
\hline
\end{tabular}

$\mathrm{T}=$ tratamento (ver definição na Tabela 1);

* Médias não seguidas pela mesma letra, na linha, diferem significativamente pelo teste de Tukey a $5 \%$.

O acúmulo de material observado na área deste experimento não é suficiente para prejudicar a formação de afilhos, indicando que, nestas condições de resíduo, a queima não é justificada e nem possível, porque o fogo não se alastra espontaneamente.

\section{Temperatura do solo}

A temperatura do solo variou de $20,0^{\circ} \mathrm{C}$ a $22,0^{\circ} \mathrm{C}$, na superfície, de $15,9^{\circ} \mathrm{C}$ a $15,3^{\circ} \mathrm{C}$, a $5 \mathrm{~cm}$ de profundidade e de $14,6^{\circ} \mathrm{C}$ a $14,8^{\circ} \mathrm{C}$, a $10 \mathrm{~cm}$ de profundidade, respectivamente antes e após a queima de $22 / 06 / 93$. Não foram observadas alterações significativas com o fogo, indicando que a combustão foi de intensidade baixa. Aumentos consideráveis na tempe- ratura ao nível do solo, na maioria das situações, são esperados durante a queima de pastagens, o que pode afetar a flora e a fauna edáfica. Neste experimento, no entanto, o resíduo da pastagem observado no momento da queima (em torno de $2000 \mathrm{~kg} / \mathrm{ha}$ de MS), que é bem inferior aos resíduos das queimadas praticadas nas propriedades, produziu um fogo de baixa intensidade, e a distribuição irregular do combustível determinou uma queima em mosaico.

\section{Mesofauna do solo}

Os componentes da mesofauna edáfica identificados nas coletas estão apresentados na Tabela 3. A discussão, no entanto, será concentrada em colêmbolos e ácaros (Tabela 4), por serem numericamente mais expressivos e servirem como indicadores da condição biológica do solo em estudo, pela sua sensibilidade às alterações ambientais. Os outros grupos de animais (Tabela 5) foram apresentados em caráter ilustrativo, com o objetivo de dar conhecimento da composição mesofaunística da pastagem natural, sendo uma informação complementar importante, porque existe grande carência na literatura gaúcha, nesta área.

Tabela 3. Componentes da mesofauna identificados em uma pastagem natural. UFSM, Santa Maria, RS (1992/ 1994).

Filo Anelida

Filo Arthropoda

Classe Arachnida

Ordem Acarina

Ordem Aranea

Ordem Phalangida

Ordem Scorpionida

Classe Chilopoda

Classe Collembola

Classe Crustacea

Classe Diplopoda

Classe Insecta

Ordem Blattodea

Ordem Coleoptera

Ordem Dermaptera

Ordem Diptera

Ordem Hemiptera

Subordem Heteroptera

Subordem Homoptera

Ordem Hymenoptera

Ordem Isoptera

Ordem Lepidoptera

Ordem Orthoptera

Ordem Thysanoptera

Ordem Thysanura 
Tabela 4. Número (média de nove trampas por tratamento) de colêmbolos e ácaros capturados em uma pastagem natural submetida aos efeitos do fogo e pastejo. UFSM, Santa Maria, RS.

\begin{tabular}{lcccccc}
\hline $\begin{array}{l}\text { Datas da retirada } \\
\text { das trampas }\end{array}$ & \multicolumn{3}{c}{ Colêmbolos } & \multicolumn{3}{c}{ Ácaros } \\
do campo & $T_{1}$ & $T_{2}$ & $T_{3}$ & $T_{1}$ & $T_{2}$ & $T_{3}$ \\
& & & & & & \\
\hline
\end{tabular}

Queima de junho

\section{$14 / 06 / 93$}

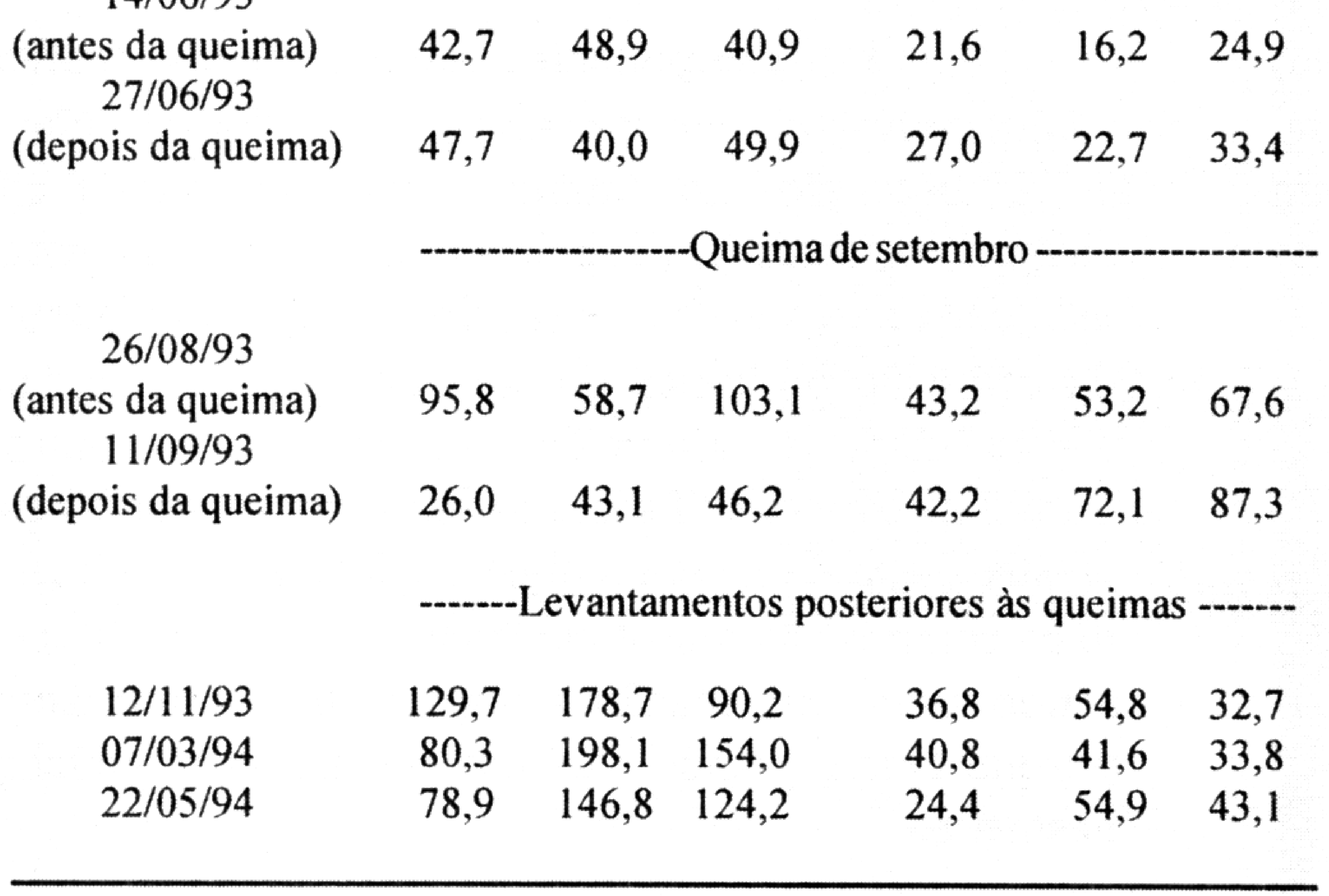

$\mathrm{T}=$ tratamento (ver descrição na Tabela 1$)$;

Diferenças não significativas quando comparadas as médias na linha (entre tratamentos e dentro da mesma época) e na coluna (dentro de tratamento, comparando apenas a coleta anterior e posterior a cada queima). segundo uma revisão de literatura conduzida por AHLGREN (1974). WALLACE (1961) observou que, aumentando a intensidade do fogo, a população de ácaros é reduzida. OLIVEIRA \& FRANKLIN (1993) verificaram que o fogo ocasionou o aumento de subordens predadoras, mas diminuiu outras subordens de Acari. MAFRA et al. (1993) encontraram uma redução na população de ácaros com o fogo. KERSTING (1994) constatou, também, uma redução na população de ácaros, embora somente oito meses após a queima. No presente trabalho, no entanto, isso não foi confirmado.

A baixa intensidade do fogo pode ter sido determinante para a ausência de efeitos significativos dos tratamentos sobre a população de ácaros e colêmbolos. É possível, também, que tenha ocorrido efeito de borda, em função do reduzido tamanho das parcelas queimadas, permitindo a fuga de alguns animais durante o fogo, bem como o rápido repovoamento das áreas queimadas a partir de áreas não queimadas. A queima em mosaico, por sua vez, possibilita que muitos animais sobrevivam, procurando refúgio nos locais remanescentes, o que também pode contribuir para a rápida recolonização das áreas queimadas.

O pastoreio não exerceu efeitos sobre colêmbolos e ácaros neste experimento, embora seja outro fator que pode influenciar muito a fauna edáfica ao alterar a vegetação e o solo. O pastejo de áreas recentemente queimadas, conforme KERSTING (1994), pode intensificar os efeitos da queima, pela contínua remoção do rebrote, mantendo o solo com reduzida cobertura vegetal, além de afetar a velociade de formação e acúmulo de material morto. KING \& HUTCHINSON (1976) observaram uma redução no número de ácaros e colêmbolos com o aumento na lotação de ovelhas. MORRIS (1968) comprovou que o pastejo intensivo reduz o número de nichos ecológicos para invertebrados, ao remover determinadas estruturas das plantas ou ao impedir o seu desenvolvimento completo.

Estudos sobre a mesofauna do solo variam em método de amostragem, habitat, tempo de observação e intensidade do fogo (AHLGREN, 1974). Duas generalizações, no entanto, segundo a autora, podem ser feitas a partir dos estudos existentes: o efeito do fogo é maior em florestas do que em pastagens e as alteraçôes ambientais posteriores à queima, via de regra, apresentam maiores efeitos que o calor do fogo
Existe consenso entre os pesquisadores sobre a redução da população de ácaros pelo fogo, 
sobre a redução das populações. A transição para condições xerófitas, a escassez de alimentos e o aumento nas flutuações da temperatura são mencionados freqüentemente, conforme revisão de AHLGREN (1974), como principais alterações no meio.

A função que o material acumulado desempenha na pastagem e no solo é bastante complexa, apresentando aspectos antagônicos. Se, por um lado, pode ser empecilho para um vigoroso rebrote, por outro, é um elemento vital para as atividades biológi-

Tabela 5. Número (média de nove trampas por tratamento) dos componentes cla mesofauna de menor expressão numérica capturados em uma pastagem natural submetida aos efeitos do fogo e pastejo. UFSM, Santa Maria, RS.

\begin{tabular}{lllllllllllllll}
\hline Componentes & $\mathrm{T}_{1}$ & $\mathrm{~T}_{2}$ & $\mathrm{~T}_{3}$ & $\mathrm{~T}_{1}$ & $\mathrm{~T}_{2}$ & $\mathrm{~T}_{3}$ & $\mathrm{~T}_{1}$ & $\mathrm{~T}_{2}$ & $\mathrm{~T}_{3}$ \\
\hline
\end{tabular}

\begin{tabular}{|c|c|c|c|c|c|c|c|c|c|}
\hline \multirow[b]{2}{*}{ Coleoptera } & \multicolumn{3}{|c|}{ (antes da queima) } & \multicolumn{3}{|c|}{$\begin{array}{l}\text { (depois da queima) } \\
\text { (de--27/06/93---.- }\end{array}$} & \multirow[b]{2}{*}{ - } & \multirow[b]{2}{*}{ - } & \multirow[b]{2}{*}{ - } \\
\hline & 0,9 & 0,4 & 14,4 & 0,3 & 0,2 & 0,9 & & & \\
\hline Orthoptera & 0,0 & 0,1 & 0,1 & 0,0 & 0,0 & 0,0 & - & - & - \\
\hline Hemiptera & 1,8 & 0,7 & 1,4 & 2,2 & 1,8 & 1,1 & - & - & - \\
\hline Hymenoptera & 8,9 & 7,6 & 13,8 & 8,8 & 4,8 & 10,4 & - & - & - \\
\hline Diptera & 3,1 & 1,4 & 3,9 & 1,7 & 1,0 & 3,2 & - & - & - \\
\hline Lepidoptera & 0,1 & 0,0 & 0,2 & 0,0 & 0,0 & 0,0 & - & - & - \\
\hline Araneae & 3,9 & 1,9 & 3,6 & 4,2 & 2,8 & 5,2 & - & - & - \\
\hline Phalangida & 1,8 & 0,8 & 2,4 & 1,1 & 0,1 & 1,0 & - & - & - \\
\hline Crustacea & 0,1 & 0,1 & 0,4 & 0,0 & 0,0 & 0,1 & - & - & - \\
\hline Isoptera & 0,8 & 0,9 & 1,6 & 0,9 & 0,7 & 1,9 & - & - & - \\
\hline Dermaptera & 0,0 & 0,0 & 0,2 & 0,4 & 0,2 & 1,1 & - & - & - \\
\hline \multirow[t]{2}{*}{ Scorpionida } & 0,1 & 0,0 & 0,0 & 0,0 & 0,0 & 0,0 & - & - & - \\
\hline & \multicolumn{3}{|c|}{ 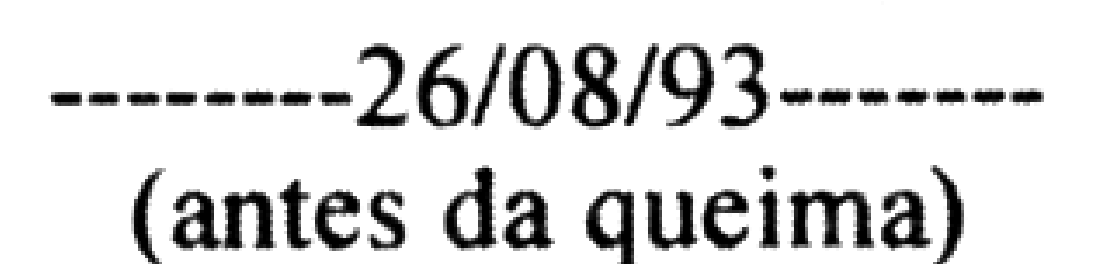 } & \multicolumn{3}{|c|}{$\begin{array}{l}\text { (depois da queima) } \\
\text {---11/09/93 }\end{array}$} & & & \\
\hline Coleoptera & 0,3 & 0,4 & 0,1 & 0,3 & 0,7 & 0,6 & - & - & - \\
\hline Orthoptera & 0,6 & 0,1 & 0,4 & 0,6 & 0,0 & 0,0 & - & - & - \\
\hline Hemiptera & 1,2 & 1,3 & 0,6 & 1,3 & 1,3 & 0,9 & - & - & - \\
\hline Hymenoptera & 5,7 & 3,6 & 5,2 & 3,8 & 3,4 & 4,6 & - & - & - \\
\hline Diptera & 1,6 & 1,1 & 1,3 & 1,3 & 1,0 & 0,7 & - & - & - \\
\hline Araneae & 1,8 & 2,4 & 2,3 & 1,1 & 2,0 & 1,0 & - & - & - \\
\hline Phalangida & 0,4 & 0,0 & 0,0 & 0,0 & 0,0 & 0,2 & - & - & - \\
\hline Isoptera & 0,6 & 0,3 & 1,2 & 0,4 & 0,4 & 0,9 & - & - & - \\
\hline \multirow[t]{2}{*}{ Dermaptera } & 0,0 & 0,1 & 0,1 & 0,6 & 0,3 & 0,3 & - & - & - \\
\hline & \multicolumn{3}{|c|}{------12/11/93------.- } & \multicolumn{3}{|c|}{ 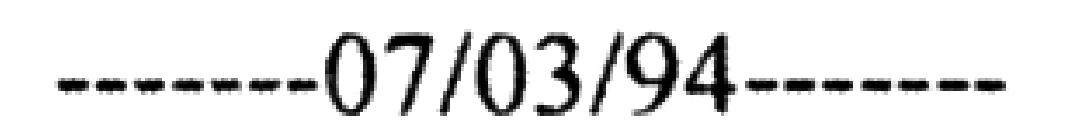 } & \multicolumn{3}{|c|}{-22/05/94-...-. } \\
\hline Coleoptera & 1,2 & 2,6 & 1,2 & 2,6 & 2,0 & 2,6 & 2,0 & 0,8 & 1,3 \\
\hline Orthoptera & 0,2 & 1,3 & 0,6 & 0,3 & 0,1 & 0,6 & 0,8 & 0,1 & 0,2 \\
\hline Hemiptera & 2,4 & 3,3 & 4,7 & 4,7 & 4,2 & 2,3 & 2,6 & 3,0 & 2,6 \\
\hline Hymenoptera & 6,4 & 11,3 & 14,7 & 19,1 & 21,8 & 40,2 & 7,3 & 10,7 & 13,6 \\
\hline Diptera & 2,9 & 4,8 & 2,4 & 2,7 & 2,4 & 3,4 & 5,1 & 4,8 & 6,4 \\
\hline Lepidoptera & 0,0 & 0,0 & 0,0 & 0,0 & 0,0 & 0,0 & 0,0 & 0,1 & 0,0 \\
\hline Araneae & 4,0 & 6,0 & 5,9 & 7,0 & 8,0 & 4,1 & 4,9 & 6,4 & 5,4 \\
\hline Phalangida & 0,0 & 0,2 & 0,0 & 0,0 & 0,0 & 0,0 & 0,0 & 0,0 & 0,0 \\
\hline Chylopoda & 0,0 & 0,0 & 0,0 & 0,1 & 0,1 & 0,1 & 0,0 & 0,0 & 0,0 \\
\hline Diplopoda & 0,0 & 0,0 & 0,0 & 0,0 & 0,0 & 0,1 & 0,0 & 0,0 & 0,0 \\
\hline Crustacea & 0,7 & 0,0 & 0,0 & 0,0 & 0,2 & 0,0 & 0,0 & 0,0 & 0,0 \\
\hline Isoptera & 0,2 & 1,0 & 1,4 & 0,6 & 0,8 & 1,3 & 2,0 & 2,0 & 2,7 \\
\hline Dermaptera & 0,2 & 0,0 & 0,4 & 0,0 & 0,1 & 0,3 & 0,1 & 0,1 & 0,2 \\
\hline Thysanura & 0,0 & 0,0 & 0,0 & 0,0 & 0,2 & 0,0 & 0,0 & 0,1 & 0,0 \\
\hline Thysanoptera & 0,1 & 0,0 & 0,0 & 0,1 & 0,1 & 0,0 & 0,4 & 0,4 & 0,1 \\
\hline Blattodea & 0,0 & 0,0 & 0,0 & 0,1 & 0,0 & 0,0 & 0,0 & 0,0 & 0,0 \\
\hline
\end{tabular}

$\mathrm{T}=$ tratamento (ver descrição na Tabela 1). cas no solo, oferecendo abrigo e servindo como alimento para os animais edáficos. A palhada serve, também, como proteção do solo contra o impacto da chuvi, vento e insolação.

\section{CONCLUSÕES}

O fogo diminui o resíduo de matéria seca da pastagem e essa redução é maior ainda com o paste $\mathrm{o}$; fogo de baixa intensidade não altera a temperatura do solo e uma queima ou duas queimas sucessivas de baixa intensidade, em pequenas áreas e em mosaico não afetam a população de colêmbolos e ácaros do solo.

\section{AGRADECIMENTOS}

Os autores agradecem a todos os colaboradores deste trabalho. Um agradecimento especial é feito a Luiz Guilherme B. Figueiredo, bolsista de Iniciação Científica $(\mathrm{CNPq})$ do Departamento de Solos da UFSM. pelo auxílio na contagem da mesofauna.

\section{REFERÊNCIAS BIBLIO- GRÁFICAS}

AHLGREN, I.F. The effect of fire on soil organisms. In: KOZLOWSKI T.T., AHLGREN. C.E. Fire and ecosystems. New York: Academic Press, 1974. p. 47-72

BARNES, R.F.. TAYLOR. T.H Grassland agriculture and ecosystem concepts. In: HEATH. M.E., BARNES, R.F., METCALFE, D.S. Forages: the science of grassland agriculture. 4. ed. Ames: Iowa State University Press, 1985. p. 12-20

BULAN, C.A., BARRETT, G.W. The effects of two acute stresses on the arthropod component of an experimental grassland ecosystem. Ecology, v. 52, n. 4, p 597-605, 1971

CORREAA, A.N.S., ARONOVICH. S Influência da queima periódica sobre a vegetação e sobre a fertilidade dos terrenos de pastagens Rev Soc Bras Zoot, Viçosa. v. 8. n. 2, p. 332-347, 1979 
COSTA, J.M.V. da, GARDNER, A.L. Sistema BOTANAL 2: manual do usuário. Brasília: EMBRAPA, 1984. 27 p.

KERSTING, C.E.B. Avaliação dos efeitos da queima, seguida de pastejo ou diferimento em uma pastagem natural. Santa Maria: UFSM, 1994. 122 p. Dissertação (Mestrado em Produção Animal) - Curso de Pós-graduação em Zootecnia, Universidade Federal de Santa Maria, 1994.

KING, K.L., HUTCHINSON, K.J. The effects of sheep stocking intensity on the abundance and distribution of mesofauna in pastures. J Appl Ecol, Oxford, v. 13, p. 41-55, 1976.

MAFRA, A.L., SANTOS, J.C.P., RHEINHEIMER, D.S. et al. Queima de campo nativo. I: Fauna edáfica. In: CONGRESSO BRASILEIRO DE CIÊNCIA DO SOLO, 24., 1993, Goiânia. Anais... Goiânia: Soc. Bras. Ciênc. Solo, 1993. p. 267-268.

MORENO, J.A. Clima do Rio Grande do Sul. Porto Alegre: Secretaria da Agricultura, 1961. 41 p.

MORRIS, M.G. Differences between the invertebrate faunas of grazed and ungrazed chalk grassland. I. Responses of some phytophagous insects to cessation of grazing. J Appl Ecol, Oxford, v. 4, p. 459-474, 1968.

OLIVEIRA, E.P. de, FRANKLIN, E. Efeito do fogo sobre a mesofauna do solo: recomendações em áreas queimadas. Pesq Agropec Bras, Brasília, v. 28, n.3, p. 357-369, 1993.

RAPOPORT, E.H. La fauna edafica y sus aplicaciones en la caracterizacion de los suelos. In: CONGRESSO LATINO-AMERICANO DE BIOLOGIA DO SOLO, 2., 1968, Santa Maria. Anais... Santa Maria: Universidade Federal de Santa Maria, Instituto de Solos e Culturas, 1968. p. 155-173.

RICHARDS, B.N. Introduction to the soil ecosystem. London: Longman, 1978. $266 \mathrm{p}$.

TOTHILL, J.C., HARGREAVES, J.N.G., JONES, R.M. BOTANAL: a comprehensive sampling and computing procedure for estimating pasture yield and composition. I. Field sampling. Trop Agron Techn Memor, Queensland, n. 8, p. 1-20, 1978

TRETZEL, E. Technik und bedeutung des fallenfanges für ökologische untersuchungen. Zool Anz, v. 155, p. 276-287, 1955.

VOGL, R.J. Effects of fire on grasslands. In: KOZLOWSKI, T.T., AHLGREN, C.E. Fire and ecosystems. New York: Academic Press, 1974. p. 139-194.

WALLACE, M.M.H. Pasture burning and its effect on the aestivating eggs of Halotydeus destructor (Tuck.). Aust J Exp Agric An Husb, Melbourne, v. 1, p. 109-111, 1961.

Ciência Rural, v. 26, n. 3, 1996. 